\title{
Hormigas cazadoras en sistemas productivos del piedemonte amazónico colombiano: diversidad y especies indicadoras
}

\author{
Maria Catalina SANABRIA-BLANDÓN ${ }^{1}$, Patricia Chacón de ULLOA²
}

\section{RESUMEN}

Para conocer la abundancia, riqueza y composición de hormigas cazadoras y su variación en los sistemas productivos del departamento de Caquetá, se seleccionaron 27 fincas representativas de tres ventanas (agroforestal, silvopastoril y tradicional), en las cuales se identificaron hasta nueve clases de usos del suelo. Las proporciones dedicadas a pastos limpios, agroforestales y rastrojos viejos, fueron las que más contribuyeron a diferenciar las ventanas. Se muestrearon cinco puntos por finca mediante cuatro métodos para captura de hormigas (TSBF, lavado de suelo con formol, escrutinio de hojarasca y búsqueda directa). De un total de 280 individuos se determinaron nueve géneros y 35 especies de las subfamilias Ectatomminae, Paraponerinae y Ponerinae; el género más diverso fue Pachycondyla con 13 especies. La abundancia de hormigas se correlacionó directamente con la riqueza de especies y con el número de especies exclusivas. La riqueza fue mayor en la ventana agroforestal (24 especies), seguida de la silvopastoril (19) y de la tradicional (8). Cinco especies fueron comunes a las tres ventanas y siete especies (Ectatomma ruidum, Hypoponera sp.1 y sp.4, Odontomachus haematodus, O. brunneus y Pachycondyla constricta) marcaron las diferencias en composición. Odontomachus brunneus resultó indicadora para la ventana tradicional y Paraponera clavata para la ventana silvopastoril. La búsqueda directa fue el método más eficiente para muestrear hormigas cazadoras en el piedemonte amazónico.

PALABRAS-CLAVE: agroforestal, silvopastoril, tradicional, muestreo de hormigas, Odontomachus brunneus, Paraponera clavata, Caquetá.

\section{Hunter ants in productive systems of Colombian amazon foothills: diversity and indicator species}

\begin{abstract}
We studied the abundance, richness and composition of hunting ants and their variation in production systems in department of Caquetá-Colombia, 27 farms were selected in three productive systems (agroforestry, silvopastoral and traditional), in this nine kinds of soil uses were identified. The proportions of clean pasture, agroforestry and old stubble contributed most to differentiating the systems. Five points were sampled per farm using four different methods for sampling ants (TSBF, formalin soil washing, screening of litter and direct search). A total of 280 organisms was collected, corresponding to nine genera and 35 species, belonging to the subfamilies Ectatomminae, Paraponerinae and Ponerinae. Pachycondyla was the most diverse genus (13 species). The abundance of ants was directly correlated with species richness and the number of exclusive species. The highest richness was observed in the agroforestry (24 species), followed by silvopastoral (19) and the lowest in traditional (8). Five species were common in the three systems and seven species marked the most differences in composition between systems (this was: Ectatomma ruidum, Hypoponera sp.1 and sp.4, Odontomachus haematodus, O. brunneus and Pachycondyla constricta). The species Odontomachus brunneus was indicator for the traditional system and Paraponera clavata was in silvopastoral system. Direct search was the most efficient method to sample ants in the Colombian Amazon foothills.
\end{abstract}

KEYWORDS: agroforestry, silvopastoral, tradicional cattle pasture, ant samples, Odontomachus brunneus, Paraponera clavata, Caquetá.

1 Universidad del Valle. catasanabria@gmail.com

2 pachacon@univalle.edu.co 


\section{INTRODUCCIÓN}

En la Amazonía la deforestación es la principal causa de pérdida de biodiversidad (Laurence et al. 2004). El piedemonte Amazónico colombiano ha sido afectado por la transformación de sus bosques para el establecimiento de sistemas productivos pecuarios (Maldonado y Velásquez 1994). En la actualidad, las condiciones económicas y sociológicas de la región determinan las prácticas de uso del suelo, los sistemas de producción y la biodiversidad, generando un complejo mosaico de fragmentos y parches de vegetación (Velásquez-Valencia 2009). Se calcula que el departamento de Caquetá ocupa el $33.4 \%$ del territorio nacional y de este el 31.2\% (aproximadamente 2'775.000 hectárea) son pasturas dedicadas al pastoreo (PRODARAM 1979). Es importante realizar estudios que permitan detectar el efecto del manejo de los suelos destinados a la ganadería sobre la biodiversidad, pues con la pérdida de macroinvertebrados asociados al suelo se afectan los procesos de degradación y mantenimiento estructural del mismo (Lavelle 1997; Blanchart et al. 1997; Chauvel et al. 1999).

Entre los insectos, las hormigas revisten gran importancia ecológica debido a que funcionan en diversos niveles dentro de un ecosistema (Majer 1983; Hölldobler y Wilson 1990), representan gran parte de la biomasa, abundancia y riqueza de especies (Forgarait 1998) además son fáciles de muestrear y su taxonomía es relativamente bien conocida, por lo que han sido usadas como indicadores ecológicos y de biodiversidad en sistemas naturales y perturbados (Lobry de Bryun 1999; Arcila y Lozano-Zambrano 2003; Andersen y Majer 2004; Hoffman y Andersen 2004).

En Colombia, varios estudios que han examinado la diversidad de hormigas cazadoras en diferentes estados sucesionales o paisajes rurales, coinciden en que hábitats de bosque son más ricos en especies, las cuales desaparecen o disminuyen su abundancia con la pérdida de cobertura vegetal natural. Así, en la región andina se estimó una reducción del $42,3 \%$ en el departamento de Antioquia (Serna y VergaraNavarro 2008), 53\% y 50\% en Caldas (Jiménez et al. 2008b; Abadía et al. 2010), 67,1\% en Quindío (Chaves et al. 2008) y $53,1 \%$ en el valle geográfico del río Cauca (Arcila et al. 2008). En contraste, no hay suficiente información sobre las hormigas cazadoras en ecosistemas ganaderos; se destacan los trabajos en sistemas silvopastoriles del Valle del Cauca (Ramírez y Enríquez 2003) y en siete usos de la tierra en los departamentos de Quindío y Valle (Rivera et al. 2008; Rivera 2009). En ambos estudios se evidencia pérdida de diversidad a medida que se intensifica el manejo ganadero.

Aunque para el departamento de Caquetá se conocen 17 géneros y 76 especies de hormigas cazadoras (Arias-Penna 2008a, 2008b; Fernández 2008)), asociadas a diferentes hábitats y pisos térmicos, no se tiene conocimiento sobre este grupo en sistemas productivos ganaderos que son la principal fuente de subsistencia para la región, seguidos de la agricultura. Este trabajo documenta la variación en la riqueza y composición de hormigas cazadoras en los sistemas agroforestal, silvopastoril y tradicional que hacen parte del paisaje de la región del piedemonte amazónico colombiano.

\section{MATERIAL Y MÉTODOS}

\section{Área de estudio}

El Departamento del Caquetá con una extensión de $88.965 \mathrm{~km}^{2}$ se localiza en el noroeste de la región Amazónica, entre los $0^{\circ} 42^{\prime} 17^{\prime \prime}$ de latitud sur y $2^{\circ} 04^{\prime} 13^{\prime \prime}$ de latitud norte y entre los $74^{\circ} 18^{\prime} 39^{\prime \prime}$ y $79^{\circ} 19^{\prime} 35^{\prime \prime}$ de longitud oeste. La precipitación media es de $3.800 \mathrm{~mm} /$ año sin estación seca bien definida; la humedad relativa es superior al $80 \%$ y la temperatura oscila entre $18^{\circ}$ y $36^{\circ} \mathrm{C}$ con una media anual de $25^{\circ} \mathrm{C}$. La zona de vida corresponde a bosque húmedo tropical (Bh-T). Prevalecen los suelos planos con altitudes entre los 200 a 400 m. (Gobernación del Caquetá 2008, Gutiérrez et al. 2004).

En la región del piedemonte se distinguen tres ventanas productivas denominadas: Tradicional, Silvopastoril y Agroforestal. Las ventanas se caracterizan por la predominancia de algunos usos del suelo que a su vez se clasifican en nueve tipos:

Pasto con leñosas: Plantaciones de pastos con arboles maderables de talla relativamente grande $(\mathrm{DAP}>10 \mathrm{~cm})$.

Pasto con arboles dispersos: Plantaciones de pastos, hay árboles o arbustos dispersos (< 100 individuos por hectárea).

Pasto limpio: Plantaciones de pasto nativo o introducido y sin árboles; corresponde a ganadería intensiva.

Rastrojo viejo: Rastrojo de al menos cuatro ańos, con arbustos y herbáceas en regeneración. Ocasionalmente árboles de caucho (Hevea brasiliensis) como evidencia de que en el pasado se cultivaba esta especie.

Rastrojo joven: Rastrojo de hasta tres años de edad con restos de cultivos abandonados.

Plantación con forrajeras nativas: Cultivos de pastos nativos e introducidos con árboles o arbustos de alto valor proteico.

Agroforestal: Pastos y gramíneas para pastoreo, bancos de proteína, caucho, frutales amazónicos y árboles maderables nativos.

Plantación de palma: Monocultivo de Palma africana (Elaeis guineensis).

Sitio familiar: También llamado huerto habitacional con cultivos de "pan coger" como leguminosas (fríjol), hortalizas (tomate), aromáticas y especias, entre otros. 


\section{Obtención y procesamiento de muestras}

El trabajo de campo se realizó durante el mes de Junio de 2008. En cada ventana productiva se seleccionaron nueve fincas y en cada finca se escogieron al azar cinco puntos, separados entre sí por una distancia aproximada de $230 \mathrm{~m}$, para un total de 135 puntos de muestreo en toda el área de estudio. Para la colecta de hormigas se aplicó el siguiente protocolo en cada punto de muestreo (Figura 1): 1) Método de TSBF (Tropical Soil Biology and Fertility) (Lavelle 1988, Anderson y Ingram 1993), que consistió en marcar un monolito central de $25 \mathrm{~cm}$ de lado y examinar en tres estratos (a nivel del suelo hasta $20 \mathrm{~cm}$ de profundidad); 2) Cinco metros al norte (MAD N) y cinco metros al sur (MAD S) se hizo escrutinio de hojarasca y suelo en un área de $1 \mathrm{~m}^{2}$ y hasta una profundidad de $10 \mathrm{~cm}$.; 3) Cinco metros al este (FORM E) y cinco metros al oeste (FORM O), se marcó un cuadro de $50 \mathrm{~cm}$ de lado al cual se agregó aproximadamente dos litros de formol al 5\%; 4) Búsqueda directa durante 10 minutos/ hombre alrededor del área muestreada. En total el esfuerzo de muestreo comprendió 135 monolitos, 270 muestras de hojarasca, 270 muestras tratadas con formol y 1350 horas/ hombre de búsqueda directa. Todas las hormigas cazadoras se determinaron a género (Bolton 1994; Fernández y Palacio 2003) y el 60\% a especie (Brown 1977; Lattke 1990; Lacau y Delabie 2002; Longino 2008; Mackay y Mackay 2008); los especímenes se compararon con la colección del Museo de Zoología de la Universidad de Sao Paulo (MZUSP) y luego se preservaron en el museo de Entomología de la Universidad del Valle (MUSENUV).

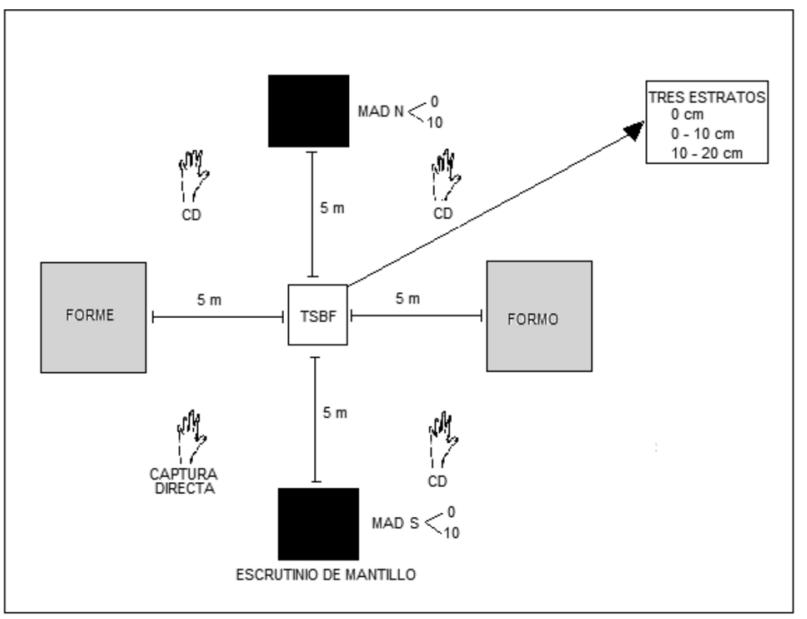

Figura 1 - Métodos utilizados para el muestreo de hormigas. TSBF: Monolito de suelo; MAD N y MAD S: Escrutinio de mantillo; FORM E y FORM 0: Lavado de suelo con formol; CD: Captura directa.

\section{Análisis de los datos}

Para comparar la abundancia y riqueza promedio de especies entre las tres ventanas productivas, se aplicó un análisis de Kruskall-Wallis (PAST). Se buscaron correlaciones (coeficiente de Pearson) (Moreno 2001; Hammer et al. 2001) entre la abundancia de hormigas cazadoras, la riqueza de especies y el número de especies exclusivas por ventana productiva. Para encontrar diferencias en la composición de usos del suelo entre ventanas, se utilizó el ANOSIM (Analysis of Similarities) que pone a prueba la hipótesis de no diferencia entre grupos (usos del suelo) (Quinn y Keough 2002). Luego se calcularon porcentajes de disimilitud (SIMPER) (PAST) (Hammer et al. 2001; Searby et al. 2004) para establecer cuales usos del suelo contribuyen a diferenciar las ventanas del paisaje. Los mismos análisis se aplicaron para encontrar diferencias en la composición de hormigas entre ventanas productivas.

Entre las hormigas cazadoras se buscaron especies indicadoras mediante el método de Dufrene y Legendre (1997) basado en el grado de especificidad y de fidelidad de una especie a un hábitat (ventana productiva). Este método ha sido utilizado para hormigas cazadoras en paisajes ganaderos (Abadía et al. 2010). El análisis de especies indicadoras (IndVal) arroja valores que van desde 0 (no indicador) hasta 100 (indicador perfecto); las especies que obtienen un alto valor son las mejores indicadoras ya que poseen una mayor probabilidad de ser detectadas, a diferencia de las especies raras (Dufrene y Legendre 1997). Finalmente mediante simulaciones de Monte Carlo se probó la hipótesis nula de que los valores de indicación observados no son mayores que los esperados por el azar (PC-ORD v. 4.34) (Mc Cune y Grace 2002).

\section{RESULTADOS}

\section{Generalidades del ensamblaje}

Se capturaron 280 hormigas cazadoras de tres subfamilias, nueve géneros y 35 especies (Tabla 1). El género más diverso fue Pachycondyla (13 especies) seguido de Hypoponera (5), Odontomachus, Gnamptogenys y Ectatomma con el mismo número de especies (4). Las ponerinas Hypoponera sp.1, Odontomachus haematodus y O. brunneus; y la llamada hormiga cachona Ectatomma ruidum, contribuyeron con el 56.3\% de la abundancia total.

Los valores totales de abundancia, riqueza y exclusividad de especies fueron mayores en la ventana agroforestal, seguida de la ventana silvopastoril y por último de la ventana tradicional (Tabla 1). Se hallaron diferencias significativas en la abundancia promedio $(\mathrm{H}=6.685 ; \mathrm{P}=0.035)$ y en la riqueza promedio $(\mathrm{H}=13.04 ; \mathrm{P}=0.001)$ entre ventanas (Figura 2$)$. El número de especies exclusivas por ventana se correlacionó directamente con la abundancia $(\mathrm{r}=0,987, \mathrm{P}<0,05)$ y con la riqueza $(\mathrm{r}=0,980, \mathrm{P}<0,05)$. Por otro lado, seis especies $E$. ruidum, E. tuberculatum, Hypoponera sp.1, O. haematodus, $O$. 
Tabla 1 - Abundancia de las especies de hormigas cazadoras recolectadas en tres ventanas productivas del departamento de Caquetá. Métodos de Captura: 1.TSBF, 2. Mantillo, 3. Lavado con formol, 4. Captura directa.

\begin{tabular}{|c|c|c|c|c|c|c|}
\hline Subfamilia y Especie & Método Captura & Agroforestal & Silvopastoril & Tradicional & Total & $\%$ \\
\hline \multicolumn{7}{|l|}{ Ectatomminae } \\
\hline Ectatomma lugens (Emery, 1894) & 1,2 & 3 & - & - & 3 & 1.1 \\
\hline Ectatomma ruidum (Roger, 1861) & $1,2,3,4$ & 4 & 18 & 1 & 23 & 8.1 \\
\hline Ectatomma sp.1 & 2 & 1 & - & - & 1 & 0.4 \\
\hline Ectatomma tuberculatum (Olivier,1972) & $2,3,4$ & 7 & 4 & 1 & 12 & 4.2 \\
\hline Gnamptogenys ericae (Roger, 1863) & 4 & - & 1 & - & 1 & 0.4 \\
\hline Gnamptogenys porcata (Emery, 1896) & 2,4 & 2 & - & - & 2 & 0.7 \\
\hline Gnamptogenys sp.1 & 4 & - & 5 & - & 5 & 1.8 \\
\hline Gnamptogenys sulcata (Smith, 1858) & 3 & - & 1 & - & 1 & 0.4 \\
\hline \multicolumn{7}{|l|}{ Paraponerinae } \\
\hline Paraponera clavata (Fabricius, 1775) & 4 & - & 3 & - & 3 & 1.1 \\
\hline \multicolumn{7}{|l|}{ Ponerinae } \\
\hline Anochetus sp.1 & 2 & 1 & - & - & 1 & 0.4 \\
\hline Centromyrmex alfaroi (Emery, 1890) & 4 & 3 & - & - & 3 & 1.1 \\
\hline Centromyrmex brachycola (Roger, 1861) & 1 & 2 & - & - & 2 & 0.7 \\
\hline Hypoponera sp.1 & $1,2,3,4$ & 25 & 16 & 16 & 57 & 20.1 \\
\hline Hypoponera sp.3 & 3,4 & 6 & - & - & 6 & 2.1 \\
\hline Hypoponera sp.4 & $1,2,3,4$ & 7 & 6 & - & 13 & 4.6 \\
\hline Hypoponera sp.8 & 4 & 1 & 1 & - & 2 & 0.7 \\
\hline Hypoponera sp.9 & 4 & 1 & - & - & 1 & 0.4 \\
\hline Leptogenys amazonica (Borgmeier, 1930) & 4 & - & 5 & - & 5 & 1.8 \\
\hline Odontomachus bauri (Emery, 1892) & $1,3,4$ & 7 & - & - & 7 & 2.5 \\
\hline Odontomachus brunneus (Patton, 1894) & $1,2,3,4$ & 2 & 8 & 25 & 35 & 12.4 \\
\hline Odontomachus haematodus (Linnaeus, 1758) & $1,2,3,4$ & 26 & 7 & 10 & 43 & 15.2 \\
\hline Odontomachus scalptus (Brown, 1978) & 3 & - & 1 & - & 1 & 0.4 \\
\hline Pachycondyla becculata (Brawn ms) & 1 & - & 1 & - & 1 & 0.4 \\
\hline Pachycondyla constricta (Mayr, 1884) & $1,2,3$ & 10 & 2 & - & 12 & 4.2 \\
\hline Pachycondyla crassinoda (Latreille, 1802) & $1,2,3,4$ & 9 & 6 & 1 & 16 & 5.7 \\
\hline Pachycondyla harpax (Fabricius, 1804) & $2,3,4$ & - & 4 & - & 4 & 1.4 \\
\hline Pachycondyla holmgreni (Wheeler, 1925) & 4 & - & - & 1 & 1 & 0.4 \\
\hline Pachycondyla impresa (Roger, 1861) & 3 & 1 & - & - & 1 & 0.4 \\
\hline Pachycondyla sp.1 & 1,2 & 7 & - & 1 & 8 & 2.8 \\
\hline Pachycondyla sp.2 & $1,3,4$ & 1 & 2 & - & 3 & 1.1 \\
\hline Pachycondyla sp.3 & 4 & - & 1 & - & 1 & 0.4 \\
\hline Pachycondyla sp.4 & 3 & 1 & - & - & 1 & 0.4 \\
\hline Pachycondyla sp.5 & 3,4 & 2 & - & - & 2 & 0.7 \\
\hline Pachycondyla sp.6 & 3 & 1 & - & - & 1 & 0.4 \\
\hline Pachycondyla stigma (Fabricius, 1804) & 4 & 2 & - & - & 2 & 0.7 \\
\hline Total individuos & & 132 & 92 & 56 & 280 & \\
\hline Total especies (exclusivas) & & $25(14)$ & $19(9)$ & $8(1)$ & 35 & \\
\hline
\end{tabular}




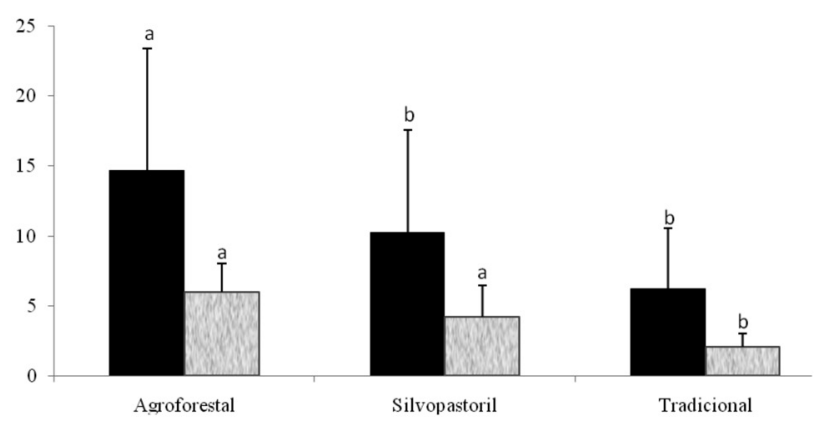

Figura 2 - Comparación de la abundancia y riqueza de hormigas cazadoras en las tres ventanas productivas. Las barras indican los valores promedio \pm la desviación estándar. El color negro representa la abundancia y color gris texturizado la riqueza.

brunneus y Pachycondyla crassinoda se registraron en las tres ventanas estudiadas.

\section{Métodos de muestreo}

La búsqueda directa resultó más eficiente en cuanto a abundancia $(34,3 \%$ de la captura total), número de especies $(65,7 \%)$ y especies exclusivas $(28,6 \%)$ (Figura 3$)$; el segundo lugar fue para la aplicación de formol al suelo seguido del escrutinio de hojarasca, mientras que con el TSBF se capturó solamente el 17,5\% de los individuos y el 37,1\% de las especies (dos de las cuales fueron exclusivas). Cinco de las seis especies comunes, a las tres ventanas, fueron recolectadas mediante los cuatro métodos.

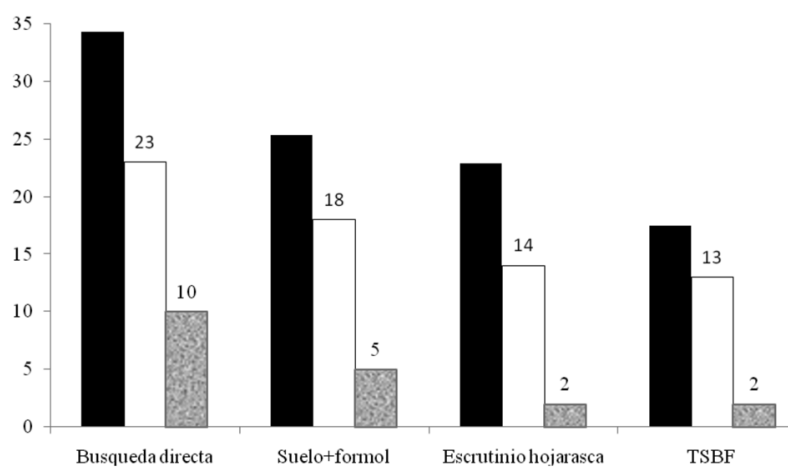

Figura 3 - Comparación de la captura de hormigas cazadoras con cuatro métodos de muestreo. En negro se observa el porcentaje (\%) de la abundancia; en blanco el número de especies recolectadas y en gris texturizado se indica el numero de especies exclusivas.

\section{Composición de usos de suelo}

El análisis de similitud mostró diferencias significativas en la composición de usos del suelo entre las tres ventanas productivas (ANOSIM R global=0.1511, P<0.0001) (Tabla 2). Las ventanas con mayores diferencias en composición fueron agroforestal y silvopastoril (84.94\%), seguidas de agroforestal y tradicional $(80.10 \%)$ y por último silvopastoril y tradicional (68.64\%). El pasto limpio fue el uso de suelo que más contribuyó a diferenciar las tres ventanas productivas; los mayores porcentajes de disimilitud se obtuvieron al comparar la ventana tradicional con la ventana agroforestal $(34,57 \%)$ o con la ventana silvopastoril (29,98\%). También el pasto limpio ocupó el primer lugar al contrastar las ventanas agroforestal y silvopastoril (20,99\%); aunque entre éstas dos ventanas, el uso de suelo denominado agroforestal aportó un valor similar $(19,70 \%)$.

Tabla 2 - Disimilitud entre tres ventanas productivas y sus usos del suelo con base en la fauna de hormigas cazadoras.

\begin{tabular}{|c|c|c|c|}
\hline Comparaciones & Uso del suelo & $\begin{array}{c}\text { Contribución } \\
(\%)\end{array}$ & $\begin{array}{c}\text { Disimilitud } \\
\text { promedio (\%) }\end{array}$ \\
\hline \multirow{9}{*}{$\begin{array}{l}\text { Agroforestal vs. } \\
\text { Silvopastoril }\end{array}$} & Pasto Limpio & 20.99 & \multirow{9}{*}{84.94} \\
\hline & Agroforestal & 19.70 & \\
\hline & Rastrojo viejo & 13.14 & \\
\hline & $\begin{array}{l}\text { Pasto con arboles } \\
\text { dispersos }\end{array}$ & 10.84 & \\
\hline & Pasto con leñosas & 7.18 & \\
\hline & Plantación forrajeras & 6.67 & \\
\hline & Rastrojo joven & 4.25 & \\
\hline & Sitio familiar & 2.17 & \\
\hline & Plantación de palma & 0 & \\
\hline \multirow{9}{*}{$\begin{array}{l}\text { Agroforestal vs. } \\
\text { Tradicional }\end{array}$} & Pasto Limpio & 34.57 & \multirow{9}{*}{80.10} \\
\hline & Agroforestal & 18.89 & \\
\hline & Rastrojo viejo & 8.543 & \\
\hline & Pasto con leñosas & 6.27 & \\
\hline & $\begin{array}{l}\text { Pasto con arboles } \\
\text { dispersos }\end{array}$ & 6.27 & \\
\hline & Rastrojo joven & 2.22 & \\
\hline & Plantación de palma & 2.22 & \\
\hline & Sitio familiar & 1.11 & \\
\hline & Plantación forrajeras & 0 & \\
\hline \multirow{9}{*}{$\begin{array}{l}\text { Silvopastoril vs. } \\
\text { Tradicional }\end{array}$} & Pasto Limpio & 29.98 & \multirow{9}{*}{68.64} \\
\hline & $\begin{array}{l}\text { Pasto con arboles } \\
\text { dispersos }\end{array}$ & 9.31 & \\
\hline & Rastrojo viejo & 8.54 & \\
\hline & Plantación forrajeras & 6.67 & \\
\hline & Pasto con leñosas & 5.26 & \\
\hline & Agroforestal & 3.33 & \\
\hline & Rastrojo joven & 2.22 & \\
\hline & Plantación de palma & 2.22 & \\
\hline & Sitio familiar & 1.11 & \\
\hline
\end{tabular}




\section{Composición de hormigas cazadoras}

Siete especies contribuyeron a la disimilitud, encontrándose diferencias significativas en su composición entre sistemas productivos (ANOSIM global $=0.03791, \mathrm{P}<0.0154$ ) (Tabla 3). La diferencia entre pares de ventanas fue similar y mostró valores muy altos, entre $91 \%$ y casi $94 \%$. Entre las ventanas agroforestal y silvopastoril, las dos especies que más aportaron a la disimilitud fueron Odontomachus haematodus (14.39\%) e Hypoponera sp. 1 (13.95\%). Entre las ventanas agroforestal y tradicional, tres especies $O$. brunneus, $O$. haematodus Hypoponera sp. 1 (18.28\% y $17.37 \%$ y 16,65 respectivamente) marcaron la diferencia. Por último, entre las ventanas silvopastoril y tradicional, también sobresalieron $O$. brunneus (20.72\%) e Hypoponera sp. 1(19.36\%).

\section{Especies indicadoras}

Solamente dos especies mostraron valor indicador significativo: $O$. brunneus resultó indicadora para la ventana

Tabla 3 - Disimilitud entre tres ventanas productivas del piedemonte amazónico colombiano con base en la fauna de hormigas cazadoras.

\begin{tabular}{|c|c|c|c|}
\hline Comparaciones & Especie & Contribución (\%) & $\begin{array}{c}\text { Disimilitud } \\
\text { promedio (\%) }\end{array}$ \\
\hline \multirow{4}{*}{$\begin{array}{l}\text { Agroforestal vs. } \\
\text { Silvopastoril }\end{array}$} & $\begin{array}{c}\text { Odontomachus } \\
\text { haematodus }\end{array}$ & 14.39 & \multirow{4}{*}{93.94} \\
\hline & Hypoponera sp. 1 & 13.95 & \\
\hline & Ectatomma ruidum & 9.17 & \\
\hline & Hypoponera sp. 4 & 7.56 & \\
\hline \multirow{4}{*}{$\begin{array}{l}\text { Agroforestal vs. } \\
\text { Tradicional }\end{array}$} & $\begin{array}{l}\text { Odontomachus } \\
\text { brunneus }\end{array}$ & 18.28 & \multirow{4}{*}{92.13} \\
\hline & $\begin{array}{l}\text { Odontomachus } \\
\text { haematodus }\end{array}$ & 17.37 & \\
\hline & Hypoponera sp. 1 & 16.65 & \\
\hline & $\begin{array}{l}\text { Pachycondyla } \\
\text { constricta }\end{array}$ & 5.23 & \\
\hline \multirow{4}{*}{$\begin{array}{l}\text { Silvopastoril vs. } \\
\text { Tradicional }\end{array}$} & $\begin{array}{c}\text { Odontomachus } \\
\text { brunneus }\end{array}$ & 20.72 & \multirow{4}{*}{91.03} \\
\hline & Hypoponera sp. 1 & 19.36 & \\
\hline & $\begin{array}{l}\text { Odontomachus } \\
\text { haematodus }\end{array}$ & 13.69 & \\
\hline & Ectatomma ruidum & 9.17 & \\
\hline
\end{tabular}

tradicional y la hormiga conga Paraponera clavata para la ventana silvopastoril; en ésta última ventana, la ponerina Pachycondyla harpax mostró un valor muy cercano a la significancia (Tabla 4).

\section{DISCUSIÓN}

\section{Generalidades del ensamblaje}

Se colectó el $53 \%$ de los géneros y el $46 \%$ de la riqueza de especies de hormigas cazadoras conocidas para el departamento de Caquetá. Seis especies no habían sido registradas (Ectatomma tuberculatum, Centromyrmex alfaroi, Leptogenys amazónica, Odontomachus scalptus, Pachycondyla becculata y P. holmgreni) (Jiménez et al. 2008a), complementando el inventario de la mirmecofauna del piedemonte amazónico.

Los métodos de colecta usados en el presente estudio exceptuando la captura directa, no se habían aplicado para efectuar muestreos rápidos de hormigas en Colombia donde es común utilizar trampas de caída y procesamiento de hojarasca en sacos winkler (Alonso 2000; Villarreal et al. 2004). Por ello, el número de especies (35) encontrado en las ventanas productivas del departamento de Caquetá es alto si se compara con registros para otras regiones como el valle geográfico del río Cauca (22 a 35 especies) (Arcila et al. 2008; Chacón de Ulloa et al. 2008), el paisaje ganadero del río Chambery en Caldas (23 a 25) (Jiménez et al. 2008b; Abadía et al. 2010); el bosque de Bremen rodeado de pastizal en Quindío (13) (Chaves et al. 2008) y algunos ecosistemas ganaderos de Quindío y Valle (23) (Rivera et al. 2008); además en los estudios citados se incluyen áreas de bosque secundario.

La abundancia y riqueza de hormigas se correlacionaron directamente y mostraron máximos valores en el sistema agroforestal, disminuyeron en el silvopastoril (30 y $24 \%$ respectivamente) y se redujeron drásticamente en el sistema tradicional ( 57 y 68\%). Esta tendencia es acorde a lo conocido en sistemas ganaderos colombianos para el mismo grupo de hormigas. Así, Ramírez y Enríquez (2003) observaron que de cinco especies colectadas en un relicto de bosque seco, solamente una o dos se encontraron en pasto estrella asociado con leucaena o con algarrobo, lo que equivale a una reducción del 60 al 80\% en la riqueza. Rivera et al. (2008) hallaron

Tabla 4 - Prueba de significancia de Monte Carlo para los máximos valores de indicación calculados en el análisis de especies indicadoras (Dufrene y Legendre 1997).

\begin{tabular}{lcccccc}
\hline \multirow{2}{*}{\begin{tabular}{l} 
Ventana \\
\cline { 2 - 6 }
\end{tabular}} & Especie & Observado & Esperado & Abundancia relativa & Frecuencia relativa & P \\
\hline Tradicional & Odontomachus brunneus & 37.2 & 11.1 & 0.12 & 0.10 & 0.001 \\
Silvopastoril & Paraponera clavata & 10.3 & 4.6 & 0.01 & 0.02 & 0.05 \\
Silvopastoril & Pachycondyla harpax & 10.3 & 4.7 & 0.01 & 0.03 & 0.057 \\
Agroforestal & Odontomachus bauri & 11.4 & 5.2 & 0.02 & 0.03 & 0.067 \\
\hline
\end{tabular}


que de 13 especies encontradas en guaduales y bosques en regeneración, solo dos estuvieron en el sistema intensivo de leucaena, sugiriendo una disminución del $85 \%$. Abadía et al. (2010) estimaron una pérdida del 53\% en la abundancia y $50 \%$ en la riqueza, en los pastizales de un paisaje subandino en Caldas.

El $40 \%$ de las especies encontradas en la ventana agroforestal fueron exclusivas de éste sistema productivo. Sobresalen Centromyrmex alfaroi y C. brachycola, dos de las tres especies conocidas en Colombia (Fernández 2008); aunque por primera vez se informa la localidad para $C$. alfaroi. Esta especie es depredadora en nidos de termitas en Brasil (Delabie 1995), lo que concuerda con su hallazgo en usos de suelo agroforestales donde hay termiteros en la base de árboles de caucho y en troncos caídos.

\section{Usos de suelo y hormigas cazadoras}

La ventana agroforestal se caracterizó por poseer una mayor proporción de usos agroforestales y rastrojos viejos, lo que podría explicar su mayor diversidad y exclusividad en hormigas cazadoras, dada la preferencia de éstas especies por hábitats con cobertura boscosa (Chacón de Ulloa et al. 2008; Chaves et al. 2008; Jiménez et al. 2008; Rivera et al. 2008; Abadía et al. 2010), que además de proveer variedad de sustratos para anidar (suelo, hojarasca y troncos caídos) (Lattke 2003; Chacón de Ulloa et al. 2008), también mantienen un microclima más constante (Jiménez et al. 2008) y probablemente ofrecen recursos alimenticios para este grupo de hormigas cuya dieta es variada (Fernández y Arias-Penna 2008); se ha observado que sistemas con árboles son más ricos en lombrices y artrópodos que aquellos sistemas que no los poseen (Chauvel et al. 1999; Molina et al. 2008). Además, el "enrastrojamiento" también atrae insectos que pueden actuar como enemigos naturales de herbívoros y parásitos de animales (Soca et al. 2002; Murgueitio y Galindo 2008), como es el caso de E. ruidum que fue reportada como depredadora natural de garrapatas en pasturas del suroccidente colombiano (Santamaría et al. 2009).

La ventana silvopastoril que se diferenció de los otros sistemas por su proporción en pasto limpio y en pasto con árboles dispersos, conservó el $40 \%$ de la riqueza del sistema agroforestal y aportó nueve especies exclusivas, una de las cuales ( $P$. clavata) mostró valor indicador. De ésta hormiga gigante se conocen sus amplios espectros de forrajeo (suelo y arbustos), alimentación (presas y néctar extrafloral) y nidificación (troncos caídos y base de árboles) (Lattke 2003) así como en zonas de cultivo y rastrojos (Arias-Penna 2008); en el presente estudio fue recolectada exclusivamente en el rastrojo viejo, pero no se puede descartar su presencia en otros usos del suelo ya que ésta especie es difícil de capturar por su gran tamańo y agresividad.
En la ventana tradicional, el pasto limpio ocupó la mayor proporción lo cual resulta en dominancia de áreas abiertas, alta exposición a la luz solar, suelos secos y compactos, condiciones que en general no favorecen el establecimiento de hormigas cazadoras (Chacón de Ulloa et al. 2008; Arcila et al. 2008), reduciéndose tanto su riqueza como la frecuencia de captura de algunas especies (Ectatomma y Pachycondyla). Sin embargo, Hypoponera sp.1 y O. haematodus no parecen afectarse; incluso $O$. brunneus triplicó su abundancia respecto al sistema silvopastoril, y mostró un alto valor indicador del sistema tradicional. Esta especie en el neotrópico se conoce de Brasil y Colombia (Fernández y Arias-Penna 2008), se la encuentra comúnmente anidando en suelo y madera en descomposición (Deyrup et al. 1988) y se adapta fácilmente a hábitats perturbados (Longino 2008).

Las especies del género Pachycondyla, el grupo que presentó la mayor riqueza, han sido asociadas a usos del suelo con vegetación arbórea. En paisajes ganaderos andinos, Rivera et al. (2008) obtuvieron 11 especies en guaduales y parches de bosque natural, mientras que solamente una especie $(P$. impressa) se halló en pasturas mejoradas con alta densidad de árboles; Ramírez y Enríquez (2003) registraron cinco especies, cuatro de las cuales fueron propias de bosques y solamente $P$. harpax se observó en el sistema silvopastoril de pasto estrella con algarrobo. También en el presente estudio, $P$. harpax fue exclusiva de la ventana silvopastoril. De manera comparable, en los sistemas productivos del Caquetá se obtuvieron 13 especies de Pachycondyla, nueve de ellas en la ventana agroforestal, seis en la silvopastoril y se redujeron a tres especies, con un solo individuo, en la ventana tradicional. $P$. crassinoda fue capturada en usos de suelo con mayor cobertura

(agroforestales y rastrojos viejos) mientras que Jiménez et al. (2008b) la registran preferiblemente en áreas abiertas.

En conclusión, los resultados obtenidos en este estudio documentan la importancia que reviste el manejo ganadero sobre la fauna de hormigas cazadoras del piedemeonte amazónico de Colombia, de modo que la ventana agroforestal, además de exhibir una mayor heterogeneidad en sus usos del suelo, combina características que favorecen una alta diversidad de hormigas con relación a los sistemas silvopastoril $\mathrm{y}$ tradicional.

\section{AGRADECIMIENTOS}

El presente estudio hizo parte del proyecto Amaz BD: "Biodiversidad de los paisajes amazónicos, determinantes socio-económicos y producción de bienes y servicios", cofinanciado por IFB-ANR bajo la gestión del Dr. Patrick Lavelle. El trabajo de campo fue apoyado por la comunidad de municipio de Florencia, el equipo de trabajo de Amaz_BD- 
Colombia y el Biólogo Carlos Santamaría. Rafael Achury y Christian Bermúdez colaboraron en la determinación taxonómica y análisis de datos. Se agradece al Dr. Roberto Brandao por permitir la consulta a la colección de hormigas del Museo de Zoología de la Universidad de Sao Paulo.

\section{BIBLIOGRAFÍA CITADA}

Abadía, J.C.; Bermúdez, C.; Lozano-Zambrano, F.H.; Chacón, P. 2010. Hormigas cazadoras en un paisaje subandino de Colombia: riqueza, composición y especies indicadoras. Revista Colombiana de Entomología, 36(1): 127-134.

Alonso, L.E. 2000. Ants as Indicators of Diversity, p. 80-88 In: Agosti, D.; Majer, J.D.; Alonso, L.E.; Schultz, T. (Eds). ANTS. Standard methods for measuring and monitoring biodiversity. Smithsonian Institution Press, London and Washington. 280 pp.

Andersen, A.N.; Majer, J.D. 2004. Ants show the way Down Under: invertebrates as bioindicators in land management. Frontiers Ecology Environment, 2(6): 291-298.

Andersen, J.P.E.; Ingram, J.S.I. 1993. Tropical Soil Biology and Fertility. A Handbook Of Methods. CAB International. Oxon. 44-46.

Arcila, C.A.; Lozano-Zambrano, F.H. 2003. Hormigas como herramienta para la bioindicación y el monitoreo. Cap. 9, págs. 159-166 In: Fernández, F. (Ed.) Introducción a las hormigas de la región Neotropical. Instituto de Investigación de Recursos Biológicos Alexander von Humboldt, Bogotá, Colombia. 398 pp.

Arcila, C.A.; Osorio, A.M.; Bermúdez, C.; Chacón de Ulloa, P. 2008. Diversidad de hormigas cazadoras asociadas a los elementos del paisaje del bosque seco, p. 531-552. In: Lozano-Zambrano, F.; Fernández, F.; Jiménez, E.; Arias, T. (Eds). Sistemática, biogeografía y conservación de las hormigas cazadoras de Colombia. Instituto de Investigaciones de Recursos Biológicos Alexander von Humboldt. Bogotá, Colombia. 617 pp.

Arias-Penna, T. 2008a. Subfamilia Ectatomminae, p. 53-107. In: Lozano-Zambrano, F.; Fernández, F.; Jiménez, E.; Arias, T. (Eds). Sistemática, biogeografía y conservación de las hormigas cazadoras de Colombia. Instituto de Investigaciones de Recursos Biológicos Alexander von Humboldt. Bogotá, Colombia. 617 pp.

Arias-Penna, T.M. 2008b. Subfamilia Paraponerinae, p. 119-122. In: Lozano-Zambrano, F.; Fernández, F.; Jiménez, E.; Arias, T. (Eds). Sistemática, biogeografía y conservación de las hormigas cazadoras de Colombia. Instituto de Investigaciones de Recursos Biológicos Alexander von Humboldt. Bogotá,Colombia. 617 pp.

Blanchart, E.; Lavelle, P.; Brandeau, E.; Le Bissonnais, Y.; Valentin, C. 1997. Regulation of soil structure by geophagous earthworm activities in humid savannas of Côte d'Ivoire. Soil Biology and Biochemestry, 149-172.

Bolton, B. 1994. Identification guide to the ant genera of the world. Harvard University Press. Cambridge, Massachusetts. 222 pp.

Brown, W.L. Jr. 1977. A supplement to the world revision of Odontomachus (Hymenoptera: Formicidae). Psyche, 83: 281-285.

Chacón de Ulloa, P.; Armbrecht, I.; Lozano-Zambrano, F. 2008. Aspectos de la ecología de hormigas cazadoras en bosques secos colombianos, p. 515-529. In: Lozano-Zambrano, F.; Fernández,
F.; Jiménez, E.; Arias, T. (Eds). Sistemática, biogeografía y conservación de las hormigas cazadoras de Colombia. Instituto de Investigaciones de Recursos Biológicos Alexander von Humboldt. Bogotá, Colombia. 617 pp.

Chauvel, A.; Grimaldi, M.; Barros, E.; Blanchart, E.; Sarrazin, M.; Lavelle, P. 1999. Pasture degradation by an Amazonian earthworm. Nature, 398: 32-33.

Chaves, M.C.; Chacón de Ulloa, P.; Lozano-Zambrano, F. 2008. Riqueza y rareza de hormigas cazadoras en el gradiente bosqueborde-pastizal de un fragmento de bosque subandino (Quindío, Colombia), p. 425-438. In: Lozano-Zambrano, F.; Fernández, F.; Jiménez, E.; Arias, T. (Eds). Sistemática, biogeografia y conservación de las hormigas cazadoras de Colombia. Instituto de Investigaciones de Recursos Biológicos Alexander von Humboldt. Bogotá, Colombia. 617 pp.

Deyrup, M.; Carlin, N.; Trager, J.C. 1988. A review of the ants of the Florida Keys. Florida Entomologist, 71(2): 163-176.

Delabie J.H.C. 1995. Inquilinismo simultâneo de duas espécies de Centromyrmex (Hymenoptera, Formicidae, Ponerinae) em cupinzeiros de Syntermes sp (Isoptera, Termitidae, Nasutermitinae). Revista brasileira de Entomología, 39:605-609.

Domínguez-Haydar Y.; Fontalvo Rodríguez, L.; Gutiérrez-Moreno L. C. 2008. Composición y distribución espacio-temporal de las hormigas cazadoras (Formicidae: grupos Poneroide y Ectatomminoide) en tres fragmentos de bosque seco tropical del departamento del Atlántico, Colombia, p. 597-528. In: Lozano-Zambrano, F.; Fernández, F.; Jiménez, E.; Arias, T. (Eds). Sistemática, biogeografía y conservación de las hormigas cazadoras de Colombia. Instituto de Investigaciones de Recursos Biológicos Alexander von Humboldt. Bogotá, Colombia. 617 pp.

Dufrenne, M.; Legendre, P. 1997. Species assemblages and indicator species: the need for a flexible asymmetrical approach. Ecological Monographs, 67(3): 45-366.

Fernández, F. 2008. Subfamilia Ponerinae s.str, p. 123-218. In: Lozano-Zambrano, F.; Fernández, F.; Jiménez, E.; Arias, T. (Eds). Sistemática, biogeografía y conservación de las hormigas cazadoras de Colombia. Instituto de Investigaciones de Recursos Biológicos Alexander von Humboldt. Bogotá, Colombia. 617 pp.

Fernández F.; Arias-Penna T. M. 2008. Las hormigas cazadoras en la región Neotropical, p. 3-39. In: Lozano-Zambrano, F.; Fernández, F.; Jiménez, E.; Arias, T. (Eds). Sistemática, biogeografia y conservación de las hormigas cazadoras de Colombia. Instituto de Investigaciones de Recursos Biológicos Alexander von Humboldt. Bogotá, Colombia. 617 pp.

Fernández, F; Palacio, E. E. 2003. Sistemática y Filogenia de las Hormigas: Breve Repaso a Propuestas, p. 29-40. In: Fernández F. (Ed). 2003. Introducción a las Hormigas de la región Neotropical. Instituto de Investigación de Recursos Biológicos Alexander von Humboldt, Bogotá, Colombia. 398 pp.

Folgarait, P. J. 1998. Ant biodiversity and its relationship to ecosystem functioning: a review. Biodiversity and Conservation, 7: 1221-1244.

Gobernación del Caquetá. 2008. (http://www.gobernaciondecaqueta. gov.co) Acceso 05/11/2008. 
Gutiérrez, R.F.; Acosta, L.E.; Cardona C.A. 2004. Perfiles Urbanos en la Amazonía Colombiana: Un enfoque para el desarrollo sostenible. Ministerio de Ambiente, Vivienda y Desarrollo Territorial; Instituto Colombiano para el Desarrollo de la Ciencia y la Tecnología "Francisco José de Caldas" Colciencias. Editor. Guadalupe Ltda. Bogotá, , Colombia. 258 pp.

Hammer, O.; Harper, D.A.T.; Ryan, P.D. 2001. PAST. Paleontological Statics software package for education and data analysis. Paleontologia Electronica, 4(1):9.

Hoffman, B.; Andersen, A. N. 2004. Responses of ants to disturbance in Australia, with particular reference to functional groups. Austral Ecology, 28: 444-46.

Hölldobler, B.; Wilson, E.O. 1990. The Ants. Harvard University Press. Springer-Verlag. Berlin, Heidelberg. 732 pp.

Jiménez, E.; Lozano-Zambrano, F.; Rodríguez, J.; Ramírez, D. 2008a. Conservación de hormigas cazadoras: rareza y endemismo, p. 407-421. In: Lozano-Zambrano, F.; Fernández, F.; Jiménez, E.; Arias, T. (Eds). Sistemática, biogeografía y conservación de las hormigas cazadoras de Colombia. Instituto de Investigaciones de Recursos Biológicos Alexander von Humboldt. Bogotá, Colombia. 617 pp.

Jiménez, E.; Lozano-Zambrano, F.; Alvaréz-Saa, G. 2008b. Diversidad alfa $(\alpha)$ y beta $(\beta)$ de hormigas cazadoras de suelo en tres paisajes ganaderos de los andes centrales de Colombia, p.439-459. In: Lozano-Zambrano, F.; Fernández, F.; Jiménez, E.; Arias, T. (Eds). Sistemática, biogeografia y conservación de las hormigas cazadoras de Colombia. Instituto de Investigaciones de Recursos Biológicos Alexander von Humboldt. Bogotá, Colombia. 617 pp.

Lacau, S.; Delabie, J.H.C. 2002. Description de trois nouvelles espéces d’Amblyopone avec quelques notes biogéographiques sur le genre au Brésil (Formicidae, Ponerinae). Bulletin de la Sociéte entomologique de France, 107: 33-41.

Lattke, J.E. 1990. Revisión del género Gnamptogenys Mayr para Venezuela. Acta Terramaris, 2: 1-47.

Lattke, J.E. 2003. Subfamilia Ponerinae, p. 261-276. In: Fernández, F. (Ed). Introducción a las hormigas de la región neotropical. Instituto de Investigación de recursos Biológicos Alexander von Humboldt. Bogotá, Colombia. 423 pp.

Laurence, W.; Albernaz, A.; Fearnside, P.; Vasconcelos, H.; Ferreira L. 2004. Deforestation in Amazonía. Science, 304: 1109.

Lavelle, P. 1988. Earthworms activities and soil system, Biology and Fertility of soil, 237-251. In: Pankhurst,C.; Doube, B.M.; Gupta, V.V.S.R. (Eds). Biological Indicators of soil Health. Cab International, Oxon, UK. 266 pp.

Lavelle, P. 1997. Faunal activities and soil processes: Adaptative strategies that determine ecosystem function. Advances in Ecological Research, 27:93-132

Lobry de Bryun, L.A. 1999. Ants as bioindicators of soil function in rural environments. Agriculture, Ecosystems and Environment, 74:425-441.

Longino, J. 2008. Hormigas de Costa Rica. (http://academic. evergreen.edu/projects/ants/Genera) Acceso: 10/11/08.
Mackay, W.; Mackay, E. 2008. Subfamilies of living ants in the new world. (http://www.utep.edu/LEB/antgenera.htm) Acceso: 05/11/08.

Majer, J. D. 1983. Ants: Bio-Indicators of Minesite Rehabilitation, Land-Use, and Land Conservation. Environmental Management, 7 (4):375-383.

Maldonado, G.; Velásquez, J. 1994. Determinación de la capacidad de carga y la ganancia de peso de Bovinos en pastoreo de Gramíneas del piedemonte amazónico de Colombia. Pasturas Tropicales, 16(2):2-8.

McCune, B.; Grace J.B. 2002. Analysis of Ecological communities. MjM Software design. Gleneden Beach, Oregon, USA. 300 pp.

Molina, C.H.; Molina, C.H.; Molina, J.E.; Molina, J.P. 2008. Carne, leche y mejor ambiente en el sistema silvopastoril intensivo con Leucaena leucocephala (Lam.) de Wit MIMOSACEAE, p. 41-65. In: Murgueitio, E.; Cuartas, C.; Naranjo, J. (Eds). Ganadería del Futuro. Investigación para el desarrollo. Fundación Cipav. Cali, Colombia. 490 pp.

Moreno, C. E. 2001. Manual de métodos para medir la biodiversidad. Universidad Veracruzana. México. 49 pp.

Murgueitio, E.; Galindo, W.F. 2008. Reconversión ambiental de fincas ganaderas en los andes centrales de Colombia, p. 67-85. In: Murgueitio, E.; Cuartas, C.; Naranjo, J. (Eds). Ganadería del Futuro. Investigación para el desarrollo. Fundación Cipav. Cali, Colombia. 490 pp.

PRODARAM. 1979. Proyecto Radargrametrico Amazonicot. La Amazonia colombiana y sus recursos. IGAC - CIAF MINDEFENSA. Santafé de Bogotá, Colombia. 590 pp.

Quinn G. P.; Keough, M. J. 2002. Experimental Design and Data Analysis for Biologists. Cambridge University Press, New York, U.S.A. 557 pp.

Ramírez, M.; Enríquez, M.L. 2003. Importance and diversity of ants in silvopastoral systems in the Cauca Valley, Colombia. Livestock Research for rural Development. Available from: http:// www.cipav.org.co/lrrd/ /rrd15/1/rami151.htm.

Rivera, L. 2009. Diversidad de hormigas y aves con relación al componente arbóreo en el agropaisaje ganadero del río La Vieja. Disertación de tesis de maestría en Ciencias-Biología. Universidad del Valle. Cali, Colombia. 117 pp.

Rivera, L.; Botero, M.; Escobar, S.; Armbrecht, I. 2008. Diversidad de hormigas en sistemas ganaderos, p. 228-243. In: Murgueitio, E.; Cuartas, C.; Naranjo, J. (Eds). Ganadería del Futuro. Investigación para el desarrollo. Fundación Cipav. Cali, Colombia. 490 pp.

Santamaría, C.; Armbrecht, J.; Lachaud, J. P. 2009. Nest distribution and food preferences of Ectatomma ruidum (Hymenoptera: Formicidae) in shaded and open cattle pastures of Colombia. Sociobiology, 53(2b): 517-537.

Searby, R. M. H.; Henderson, P. A.; Prendergast, J. 2004. Community analysis package v 3.01. Pisces Conservation Ltd. Hampshire UK.

Serna F.; Vergara-Navarro, E.V. 2008. Hormigas cazadoras de Porce (Antioquia, Colombia), p. 553-472. In: Lozano-Zambrano, F.; Fernández, F.; Jiménez, E.; Arias, T. (Eds). Sistemática, biogeografía y conservación de las hormigas cazadoras de Colombia. 
Instituto de Investigaciones de Recursos Biológicos Alexander von Humboldt. Bogotá, Colombia. 617 pp.

Soca, M.; Simón, L.; Sánchez, S.; Gómez, E. 2002. Dinámica parasicológica en Bostas de Bovinos bajo condiciones silvopastoriles. Agroforestería de las Américas, 9: 33-42

Velásquez-Valencia, A. 2009. Estructura de la comunidad de aves en sistemas de producción del piedemonte amazónico. Disertación de tesis de maestría en Ciencias-Biología. Universidad Nacional de Colombia. Bogotá, Colombia. 51 pp.
Villareal, H.; Álvarez, M.; Córdoba, S.; Escobar, F.; Fagua, G.; Gast, F.; Mendoza, H.; Ospina, M.; Umaña, A. M. 2004. Manual de métodos para el desarrollo de Inventarios de biodiversidad. Programa de inventarios de biodiversidad. Instituto de investigación de Recursos Biológicos Alexander von Humboldt. Bogotá, Colombia. 236 pp.

Recebido em 05/08/2010

Aceito em 19/10/2010 